\title{
Signal Detection for Cognitive Radios with Smashed Filtering
}

\author{
Martin Braun, Jens P. Elsner and Friedrich K. Jondral \\ Universität Karlsruhe (TH), Germany, \{braun, elsner, fj\}@int.uni-karlsruhe.de
}

\begin{abstract}
Compressed Sensing and the related recently introduced Smashed Filter are novel signal processing methods, which allow for low-complexity parameter estimation by projecting the signal under analysis on a random subspace. In this paper the Smashed Filter of Davenport et al. is applied to a principal problem of digital communications: pilot-based time offset and frequency offset estimation. An application, motivated by current Cognitive Radio research, is wide-band detection of a narrowband signal, e.g. to synchronize terminals without prior channel or frequency allocation. Smashed Filter estimation and maximum likelihood-based, uncompressed estimation for a signal corrupted by additive white Gaussian noise (Matched Filter estimation) are compared. Smashed Filtering adds a degree of freedom to signal detection and estimation problems, which effectively allows to trade signal-to-noise ratio against processing bandwidth for arbitrary signals.
\end{abstract}

\section{INTRODUCTION}

Cognitive Radio (CR) systems ${ }^{1}$ observe the radio spectrum and act upon their findings to optimize spectrum usage. The observable bandwidth is bounded by the bandwidth of the radio receiver front-end. A large processing bandwidth is preferable but increases the requirements on the analog-todigital converter (ADC) subsystem. Signal processing methods based on compressed observations of the signal can be used to increase the observation bandwidth while keeping the requirements on the $\mathrm{ADC}$ constant - although the compression is, in general, lossy when noise is present.

Compressed Sensing (CS) is a novel approach to digital data acquisition. CS theory asserts that one can gather the same information from signals by taking far less measurements than the Shannon-Nyquist-Theorem dictates, if the signal has a sparse representation in some basis [2], [3]; however, these measurements are not necessarily point samples, but some linear functionals of the signal. [4] and [5] apply the idea of CS to parameter estimation problems, in which not the signal itself has a sparse representation in some basis, but the set of all possible signals, given by all possible parametrizations, is a lower-dimensional manifold of the signal space.

Applications of CS in CR systems have been proposed before, such as identification of white spaces [6]. The application of interest here is the detection of narrow-band pilot sequences in a wide band of frequencies in order to synchronize wireless terminals without prior frequency allocation. The recently introduced Smashed Filter [4] offers a trade-off between signal-to-noise ratio and processing bandwidth, and can hence

\footnotetext{
${ }^{1}$ For an introduction to Cognitive Radio cf. [1].
}

be used to analyse a wider spectrum band without having to increase the sampling speed of the ADCs.

This paper demonstrates the effectiveness of the smashed filter pilot detection method for a QPSK modulated pilot signal corrupted by additive white Gaussian noise (AWGN).

The paper is organised as follows: Section II describes the mathematical model and provides the theoretical background for uncompressed estimation. Section III gives a short introduction to CS as needed for this paper and applies it to the estimation problem. Simulation results are presented in Section IV. Sections V and VI show an application example and conclude.

\section{Analytical Model}

Assume the bandlimited signal $p(t)$ is the baseband representation of a known, narrow-band, QPSK modulated pilot sequence. $p(t)$ is shifted to an unknown centre frequency $\nu$ and to an unknown time offset $\tilde{\tau}_{\max } \geq \tilde{\tau} \geq 0$. The transmitted signal $s(t)$ is then

$$
s(t, \nu, \tilde{\tau})=p(t-\tilde{\tau}) e^{j 2 \pi \nu t} .
$$

The signal is corrupted by AWGN of variance $\sigma^{2}$, resulting in the received signal

$$
r(t)=s(t, \nu, \tilde{\tau})+n(t), t \in[0, T] .
$$

$T$ is the length of the analysis interval, which is assumed at least as long as the sum of $\tilde{\tau}_{\max }$ and the length of $p(t) . n(t)$ is the noise signal. Next, assume that possible centre frequencies of $s(t)$ are bounded by limiting the maximum frequency shift to $|\nu|<f_{\max }$. As $p(t)$ is also bandlimited, the received signal can be expressed as a time-discrete signal

$$
r[k]=s[k, \boldsymbol{\Theta}]+n[k]=s[k-\tau] e^{j 2 \pi \nu \frac{k}{f_{S}}}+n[k] .
$$

$r[k]$ is sampled at equidistant intervals using a sampling frequency $f_{S}$ which must be more than twice as large than $f_{\max }$. The analysis interval then consists of $N=T f_{S}$ samples and the received signal can be represented as a vector $\mathbf{r}=(r[0], r[1], \ldots, r[N-1])^{T}$. The timing offset is expressed as the time shift in samples $\tau=\tilde{\tau} / f_{S}$, which is a justified approximation if the pilot signal is assumed to have a bandwidth much lower than the sampling rate. $\boldsymbol{\Theta}=(\nu, \tau)$ is the combined parameter vector which needs to be estimated. 


\section{A. Traditional Maximum-Likelihood Estimation}

Using traditional digital signal processing methods, a method to estimate $\Theta$ is maximum likelihood estimation. The Maximum Likelihood Estimate (MLE) is the value for $\Theta$ which maximises the joint conditional density probability function of the received signal $f_{R \mid \Theta}(\mathbf{r} \mid \Theta)$, also known as the likelihood function. The probability density function of the noise vector $\mathbf{n}=(n[0], n[1], \ldots, n[N-1])^{T}$ is that of an uncorrelated Gaussian noise with constant variance. As the transmitted signal $s[k, \boldsymbol{\Theta}]$ is deterministic for any given $\boldsymbol{\Theta}$, the likelihood function can be calculated as

$f_{\mathbf{R} \mid \boldsymbol{\Theta}}(\mathbf{r} \mid \boldsymbol{\Theta})=\left(\frac{1}{\sqrt{2 \pi} \sigma}\right)^{N} \exp \left(\frac{-(\mathbf{r}-\mathbf{s}(\boldsymbol{\Theta}))^{*}(\mathbf{r}-\mathbf{s}(\boldsymbol{\Theta}))}{2 \sigma^{2}}\right)$.

The logarithmic function is monotonic, hence the $\log a$ rithmic likelihood function $\ell(\Theta)=\ln f_{\mathbf{R} \mid \Theta}(\mathbf{r} \mid \Theta)$ can be maximised instead: maximisation of $\ell(\boldsymbol{\Theta})$ is equivalent to maximising

$$
\ell^{\prime}(\boldsymbol{\Theta})=-\|\mathbf{r}\|_{2}^{2}+2 \Re\left[\mathbf{r}^{\mathbf{H}} \mathbf{s}(\boldsymbol{\Theta})\right]-\|\mathbf{s}(\boldsymbol{\Theta})\|_{2}^{2} .
$$

As the first and last terms of (4) do not change ${ }^{2}$ with $\Theta$, this can be transformed to a simple rule for the MLE:

$$
\hat{\mathbf{\Theta}}_{\mathbf{M L}}=\underset{\Theta}{\operatorname{argmax}} \Re\left[\mathbf{r}^{\mathbf{H}} \mathbf{S}(\tilde{\mathbf{\Theta}})\right] .
$$

The term to be maximised is an inner product. As described manifold in literature, this can be implemented with a matched filter setup. A straightforward method to implement this algorithm is to restrict the number of possible frequencies. In this case, the signal is run through a bank of matched filters, one for every possible frequency offset.

\section{B. Phase offsets}

In practice, the received time-discrete signal is much more likely to have the form

$$
\mathbf{r}=\mathbf{s}(\boldsymbol{\Theta}) \cdot e^{j \varphi_{0}}+\mathbf{n}
$$

where $\varphi_{0}$ is an unknown phase shift. Applying the estimator (5) to this phase-shifted signal, the value which needs to be maximised is

$$
\begin{aligned}
\Re\left[\mathbf{r}^{H} \mathbf{s}(\tilde{\boldsymbol{\Theta}})\right] & =\Re\left[\left(\mathbf{s}(\boldsymbol{\Theta}) \cdot e^{j \varphi_{0}}+\mathbf{n}\right)^{H} \mathbf{s}(\tilde{\boldsymbol{\Theta}})\right] \\
& =\Re\left[e^{-j \varphi_{0}} \mathbf{s}^{H}(\boldsymbol{\Theta}) \mathbf{s}(\tilde{\boldsymbol{\Theta}})+\mathbf{n}^{H} \mathbf{s}(\tilde{\boldsymbol{\Theta}})\right] \\
& =\Re\left[e^{-j \varphi_{0}}(\underbrace{\mathbf{s}^{H}(\boldsymbol{\Theta}) \mathbf{s}(\tilde{\boldsymbol{\Theta}})}_{\tilde{C}_{s}}+\underbrace{\mathbf{n}^{\prime H} \mathbf{s}(\tilde{\boldsymbol{\Theta}})}_{\tilde{C}_{e}})\right] .
\end{aligned}
$$

$\mathbf{n}^{\prime}=e^{j \varphi_{0}} \mathbf{n}$ is a rotated version of the noise.

Due to the unknown phase offset, (7) will not be maximal for $\tilde{\boldsymbol{\Theta}}=\boldsymbol{\Theta}$ with high probability due to the rotation by $\varphi_{0}$. To

\footnotetext{
${ }^{2}$ The latter term is equivalent to the transmitted energy of $s$, which is invariant towards time- or frequency shifting.
}

solve this problem without having to estimate the phase offset, one can maximise the absolute value of the inner product instead of the real value, which eliminates the factor $e^{-j \varphi_{0}}$. One can show that $\left|\mathbf{r}^{H} \mathbf{S}(\tilde{\boldsymbol{\Theta}})\right|$ has in average the same, unique maximum as (7). In a nutshell, this is done by finding the maximum for the absolute value of $\tilde{C}_{s}$. Applying the triangle inequality to the summation notation of $\left|\tilde{C}_{s}\right|$ yields

$$
\begin{aligned}
\left|\tilde{C}_{s}\right| & =\left|\sum_{k=0}^{N-1} p^{*}[k-\tau] p[k-\tilde{\tau}] e^{j 2 \pi \frac{\tilde{\nu}-\nu}{f_{S}} k}\right| \\
& \leq \sum_{k=0}^{N-1}\left|p^{*}[k-\tau] p[k-\tilde{\tau}]\right|
\end{aligned}
$$

which is maximal for $\tau=\tilde{\tau}$ as $p[k]$ is a finite sequence. Inserting this into $\tilde{C}_{s}$ and applying the triangle inequality again returns

$$
\begin{aligned}
\left|\tilde{C}_{s}\right|_{\tau=\hat{\tau}} & =\left.\left|\sum_{k=0}^{N-1}\right| p[k-\tau]\right|^{2} e^{j 2 \pi \frac{\hat{\nu}-\nu}{f_{S}} k} \mid \\
& \leq \sum_{k=0}^{N-1}|p[k-\tau]|^{2} .
\end{aligned}
$$

Summations of type $\sum_{k} e^{j 2 \pi \alpha k}$ are maximal when $\alpha \in \mathbb{Z}$. In this case, the inequality becomes an equality for $\nu=\tilde{\nu}$. Assuming ergodicity, the error term $\tilde{C}_{e}$ does not affect the maximisation of the sum $\left|\tilde{C}_{s}+\tilde{C}_{e}\right|$ as noise and signal are not correlated. Therefore, the estimator using the absolute value has the same expected value for its maximum as the MLE, but is invariant to phase offsets. The rule for the phase invariant estimate is thus

$$
\hat{\boldsymbol{\Theta}}_{P I}=\underset{\Theta}{\operatorname{argmax}}\left|\mathbf{r}^{\mathbf{H}} \mathbf{S}(\tilde{\boldsymbol{\Theta}})\right| .
$$

\section{Compressed SENSING AND ClassificATION}

In $\mathrm{CS}$, the data used for analysis or further processing is not a signal sampled at regular intervals as known from traditional digital signal processing, but rather a collection of inner products of this signal with some compression functions. A simplified mathematical description is as follows: as described above, $\mathbf{r}=(r[0], r[1], \cdots, r[N-1])^{T}$ is a vector of $N$ samples representing the received signal, obtained through regular sampling. The compressed data set is a vector $\mathbf{y}$ of length $M<N$, which is obtained by a linear operation

$$
\mathbf{y}=\boldsymbol{\Phi} \mathbf{r} .
$$

$\Phi \in \mathbb{R}^{M \times N}$ is called the measurement matrix. The compressed signal consists of $M$ inner products of the entire original signal with the rows of $\boldsymbol{\Phi}$.

At this point it is worth having a closer look at $\mathbf{r}$. Ignoring the noise for a moment, this vector, even though it is of a high dimension $N$, only has two degrees of freedom for a fixed pilot signal: time and frequency offset. As a consequence, the entirety of possible noise-free signals can be modeled as 
a 2-dimensional manifold $\mathcal{M}$. This again gives a clue about the properties of $\boldsymbol{\Phi}$ : the dimensionality reduction must not destroy the structure of this manifold; more precisely: the euclidean distance between two points on this manifold must be approximately preserved. This has been formulated as the Restricted Isometry Property (RIP) [2], which states that for a given $\varepsilon \in[0,1]$, the following statement must hold with a high probability for any two vectors $\mathbf{x}, \mathbf{y} \in \mathcal{M}$ :

$$
(1-\varepsilon) \sqrt{\frac{M}{N}} \leq \frac{\|\mathbf{\Phi} \mathbf{x}-\mathbf{\Phi} \mathbf{y}\|_{2}}{\|\mathbf{x}-\mathbf{y}\|_{2}} \leq(1+\varepsilon) \sqrt{\frac{M}{N}}
$$

This property is given for random orthoprojectors [5], i.e. random $M \times N$ matrices with orthonormal rows, and some entirely random matrices [7], provided they are sufficiently large. Here, three kinds of measurement matrices are compared,

1) Non-Uniform Subsampling (NUS) or Random Subsampling matrices which are created by taking $M$ separate rows uniformly at random from the unit matrix $\mathbf{I}_{N}$,

2) Matrices formed by sampling the i.i.d. entries $(\boldsymbol{\Phi})_{i j}$ from a $\mathcal{N}(0,1 / N)$ distribution (white Gaussian noise),

3) Matrices formed by sampling the i.i.d. entries $(\boldsymbol{\Phi})_{i j}$ from a symmetric Bernoulli distribution (entries are $\pm 1 / \sqrt{N}$ with probability $1 / 2$ each).

The implications of the choice of $\boldsymbol{\Phi}$ on the data acquisition method are straightforward: in the first case, $M$ samples are chosen at random, the rest of the signal is discarded. In the two other cases, $\mathbf{y}$ is created by calculating $M$ inner products with realisations of some random (noise) process. Hardware implementations for types 1 and 3 have been suggested in [8] and [9]. The signal can also be sampled at its Nyquist rate and then compressed to reduce the data rate, an interesting aspect for software radio signal processing, where processing bandwidth is usually limited by computational resources.

Compressed classification methods such as the smashed filter now have to address the problem of estimating parameters or classifying signals from the compressed data vector $\mathbf{y}$.

\section{A. Smashed Filter Maximum-Likelihood Estimation}

In the compressed case, the estimation must be derived from the compressively sampled vector $\mathbf{y}$ and the measurement matrix $\boldsymbol{\Phi}$. As described in [4], this results in a new maximum likelihood condition of equivalent form, a compressed version of (5):

$$
\hat{\boldsymbol{\Theta}}_{\mathbf{M L}}=\underset{\Theta}{\operatorname{argmax}} \Re\left[\mathbf{y}^{\mathbf{H}} \mathbf{\Phi} \mathbf{s}(\boldsymbol{\Theta})\right]
$$

Likewise, the compressed form of the phase invariant estimator is

$$
\hat{\boldsymbol{\Theta}}_{\mathbf{P I}}=\underset{\boldsymbol{\Theta}}{\operatorname{argmax}}\left|\mathbf{y}^{\mathbf{H}} \mathbf{\Phi} \mathbf{s}(\boldsymbol{\Theta})\right| .
$$

The inner product is now calculated in the compressed domain by the comparing $\mathbf{y}$ to an identically compressed version of the reference signal $\mathbf{s}(\boldsymbol{\Theta})$. To show this is justified, one can examine the expected value of the inner product for a fixed received signal $\mathbf{r}$ :

$$
\begin{aligned}
E\left[\mathbf{y}^{\mathbf{H}} \boldsymbol{\Phi} \mathbf{s}(\boldsymbol{\Theta}) \mid \mathbf{r}\right] & =E\left[(\boldsymbol{\Phi} \mathbf{r})^{\mathbf{H}} \boldsymbol{\Phi} \mathbf{s}(\boldsymbol{\Theta}) \mid \mathbf{r}\right] \\
& =\mathbf{r}^{\mathbf{H}} \mathbf{E}\left[\boldsymbol{\Phi}^{\mathbf{H}} \boldsymbol{\Phi}\right] \mathbf{s}(\boldsymbol{\Theta})=\alpha \mathbf{r}^{\mathbf{H}} \mathbf{s}(\boldsymbol{\Theta})
\end{aligned}
$$

The last equality results from the fact that all the $\boldsymbol{\Phi}$ chosen here have the property that $E\left[\boldsymbol{\Phi}^{H} \boldsymbol{\Phi}\right]=\alpha \mathbf{I}_{N}$, where $\alpha$ is a real, positive value, which does not affect the maximisation. As a result, in average the smashed filter setup will return the same result as the uncompressed matched filter setup. However, the projection with a random orthoprojector will reduce the SNR with high probability by approximately $20 \log \sqrt{M / N}$ due to the compaction of an $N$-dimensional space to an $M$ dimensional one [4], [5], while the noise power itself is not affected.

An advantage of the compressed estimator is its reduced complexity: for every hypothesis $\Theta$, one inner product of length- $M$ vectors has to be calculated, unlike the inner products of length $N$ for the traditional matched filter. In order to reduce the total complexity of the estimator, the product $\boldsymbol{\Phi}_{\mathbf{S}}(\Theta)$ must not cause any additional computational cost. For instance, if NUS is chosen as CS method, the compression simply consists of picking $M$ samples from the original stream and will obviously not be cost-intensive to implement.

\section{Simulation Results}

Monte Carlo-type experiments were run to confirm the theory. The following setup was used: A root-raised-cosinefiltered QPSK signal (which is a typical narrow-band signal) was used as a pilot signal at a fixed sampling rate $f_{S}$. The occupied bandwidth (which coincides with its symbol rate) is $1 / 32$ of the sampling rate. The entire length of the pilot signal (including filter flushing) is $L=320$ samples. This signal was time-shifted by a value of 0 to 319 samples and frequencyshifted onto any frequency between $-f_{S} / 2$ and $+f_{S} / 2$ on a grid of 128 equidistant frequencies. The signal-to-noise ratio is defined as

$$
\mathrm{SNR}=\frac{P_{p}}{\sigma^{2}}, P_{p}=\frac{1}{L} \sum_{l=0}^{L-1}|p[l]|^{2} .
$$

The number of samples $N$ analysed was 640 , so that the pilot signal was definitely entirely in the observed interval, but still not too large for numeric software to perform algebraic operations in reasonable time. The number of compressed samples $M$ was kept at $10 \%$ of the original number, yielding 64 compressed samples per measurement. For every SNR value, 1000 simulations were run. The only noise source was AWGN. A traditional matched filter bank setup was run to compare the results.

Figures 1 and 2 show the mean absolute timing error and the probability of correct frequency acquisition, respectively, for a fixed compression rate of $M / N=0.1$. The SNR decline is very close to the predicted $20 \log \sqrt{M / N}=-10 \mathrm{~dB}$ as mentioned in Section III-A. For low SNR values (starting at 


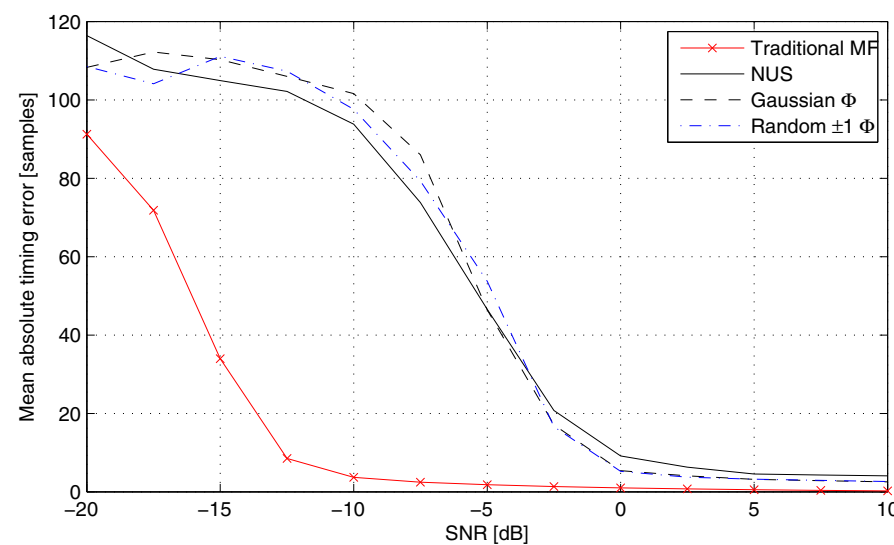

Fig. 1. Timing error for various smashed filter setups and a traditional matched filter $(\mathrm{M} / \mathrm{N}=0.1)$

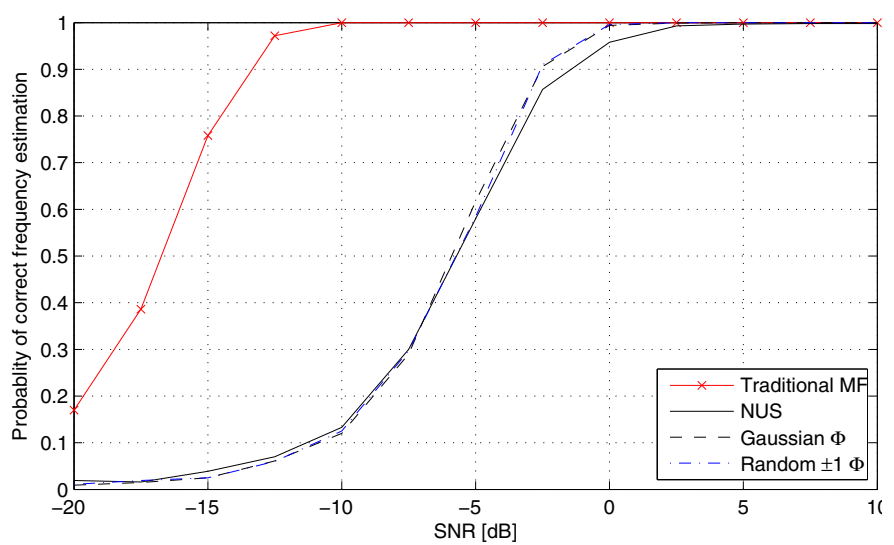

Fig. 2. Probability of correct frequency estimation $(\mathrm{M} / \mathrm{N}=0.1)$

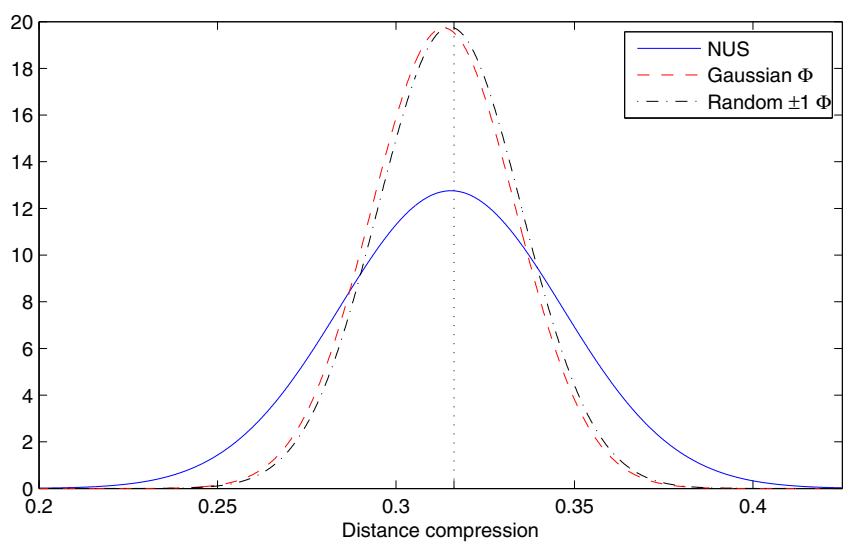

Fig. 3. Estimated probability distribution of the distance compression through dimensionality reduction

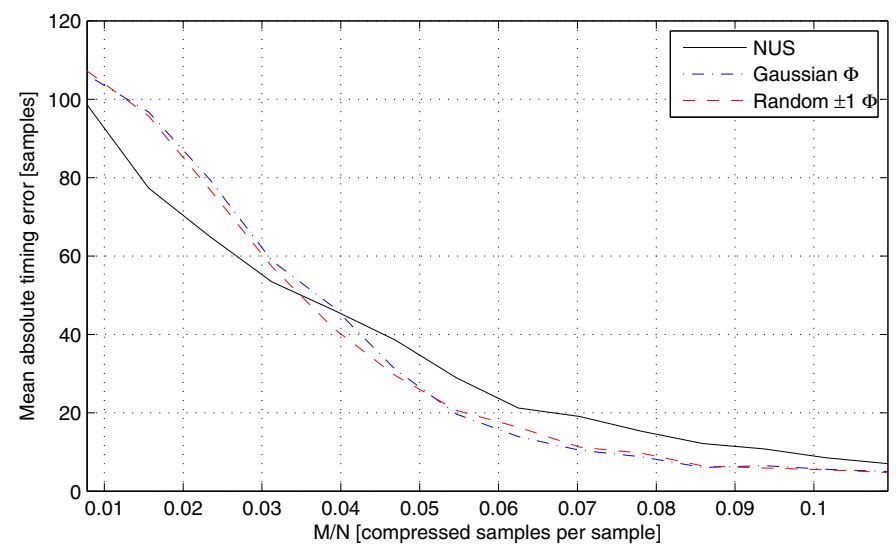

Fig. 4. Timing error for various smashed filter setups $(\mathrm{SNR}=0 \mathrm{~dB})$

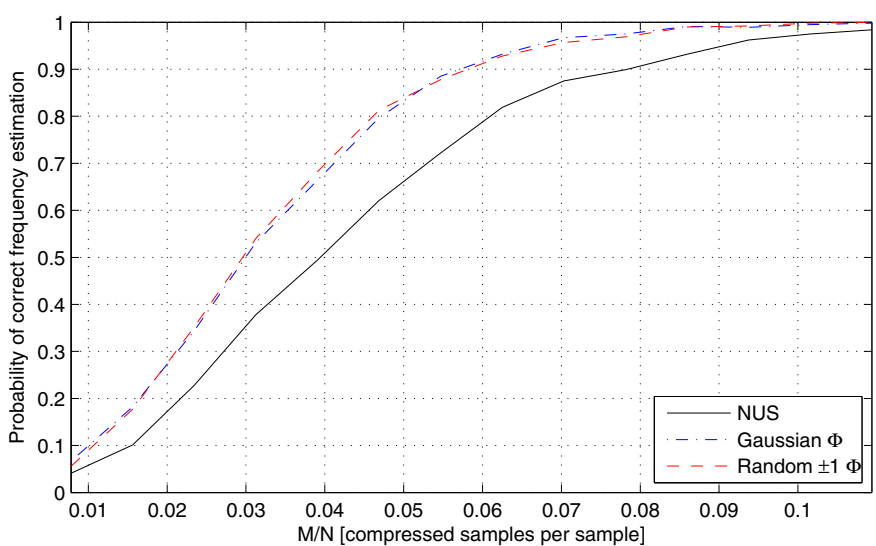

Fig. 5. Probability of correct frequency estimation $(\mathrm{SNR}=0 \mathrm{~dB})$

$-10 \mathrm{~dB})$ the timing error converges towards the worst-case timing error of 106.67 samples.

In order to estimate the quality of the different measurement matrices, the RIP was tested numerically for the same experimental setup. For all combinations $\boldsymbol{\Theta}_{1}, \boldsymbol{\Theta}_{2}$ at a fixed frequency $\nu$, the distance reductions $\left\|\mathbf{\Phi} \mathbf{s}\left(\Theta_{1}\right)-\boldsymbol{\Phi} \mathbf{s}\left(\Theta_{2}\right)\right\| / \| \mathbf{s}\left(\Theta_{1}\right)-$ $\mathbf{s}\left(\boldsymbol{\Theta}_{2}\right) \|$ were calculated for 100 random instances of $\boldsymbol{\Phi}$ and for every type of measurement matrix. From the results, the probability density functions of the distance reduction were estimated (Figure 3) by approximating the histogram with a normal distribution.

The less the distance reduction varies from $\sqrt{M / N}$ (dotted line), the better $\boldsymbol{\Phi}$ is. One can see that the NUS matrices do this worse than the other ones, which perform fairly similarly, both concerning the RIP as well as for the estimation, where NUS recognisably performs a little worse for higher SNR values.

In order to get an idea about the dimension of $M$, another set of experiments was run for $M \in[5,70]$ (yielding compression rates $M / N$ between 0.0078 and 0.1094 ). The noise level was kept at a constant SNR $=0 \mathrm{~dB}$, a level at which the traditional matched filter can still perform satisfactorily. The results are 


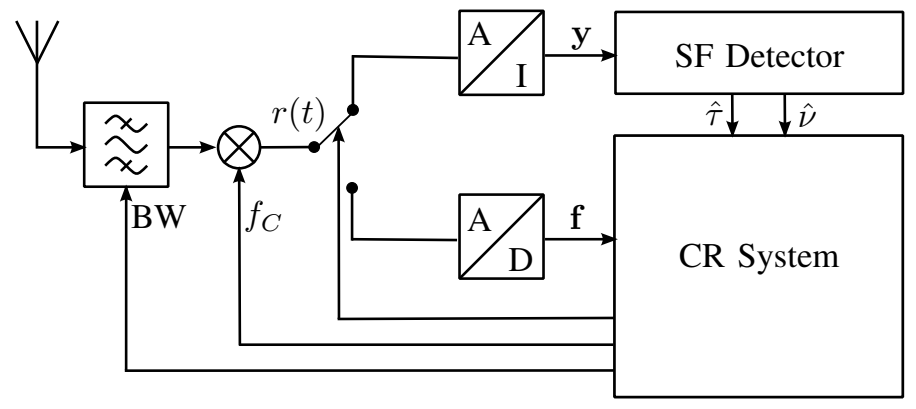

Fig. 6. Cognitive radio terminal with smashed filter signal detection

shown in Figures 4 and 5, respectively. With only $10 \%$ of the original sampling rate (cf. Figures 1 and 2), the results are already very close to those of the optimal estimator. This is consistent with the initial claim that processing bandwidth can be traded for SNR: by reducing the processing rate, SNR was reduced by $-10 \mathrm{~dB}$, reaching a level which the uncompressed matched filter can still handle.

In all cases, the estimator employed was the phase invariant estimator (16). Its performance was equivalent to that of the maximum likelihood estimator, and could tolerate random phase offsets as predicted.

\section{APPLICATION EXAMPLE: CR TERMINAL SYNCHRONISATION}

This type of smashed filter could be used to enhance the functionality of CR systems. Assume the following situation: several CR terminals are beginning to establish an ad-hoc network. At first, no CR terminal has any knowledge about which other terminals are within its range and which frequencies are not used in their vicinity. In order to establish first contact with neighbouring terminals, every terminal starts sending a beacon signal periodically on a random centre frequency and monitors the available bandwidth for the same beacon signal from other CR terminals. As soon as one CR terminal receives the beacon signal, it knows there is a CR terminal in the vicinity and can establish a point-to-point connection by the means of some handshake protocol, possibly by sending an answer signal while the beacon signal is silent.

This kind of network assembly is extremely demanding regarding the receiver. While establishing the connection, extremely high bandwidths must be observed and scanned for beacon signals. Once a connection is established, the terminals must be able to communicate on a fairly narrow bandwidth compared to the observation bandwidth during beacon detection.

Smashed filtering can be a possible solution to creating such a receiver system. Figure 6 shows a proposed schematic of a CR terminal using CS methods. While the CR is searching for beacon signals, a very high bandwidth is shifted to baseband. This high-bandwidth signal is passed to an analogto-information converter (AIC) [8], [9] which performs the compressive sampling. The compressed signal vector $\mathbf{y}$ is passed to the smashed filter subsystem, which decides if the observed bandwidth BW contains a beacon signal and at which estimated frequency $\hat{\nu}$ and time offset $\hat{\tau}$.

Once a beacon signal has been detected, the terminal can tune the receiver front-end to the desired centre frequency $f_{C}$ and bandwidth BW. The analog signal $r(t)$, which is now comparably narrow-band, can be fed to an ADC which is suited for this kind of bandwidth and processed with traditional software radio methods. Using CS, the terminal can be designed to both observe high bandwidths and reliably process small bandwidths without employing unreasonably expensive hardware.

On the downside, scanning a wide band using CS will severely reduce SNR. However, for small distances between receivers SNR can often be spared, since signal detection requires smaller SNR than demodulation in the first place.

\section{CONCLUSiON AND Future WORK}

Smashed Filter based estimation and detection allows to trade signal-to-noise ratio against processing bandwidth. In an AWGN scenario, sampling rates can be reduced significantly without reducing the reliability of the estimator and detector and without increasing its complexity. In this paper, a narrowband randomized QPSK signal was used as a pilot - open research issues are pilot design for compressed estimation and the exact influence of different $\boldsymbol{\Phi}$ on the estimation problem. Current studies have shown that Toeplitz-structured compressed matrices maintain correlation properties and thus separate the problem of finding suitable pilot signals from the compression. Future research will address the problem of combining a Smashed Filter detector with compressed spectrum estimation techniques to allow detection of narrowband pilot signals in a scenario with non-white interferers.

\section{REFERENCES}

[1] F. K. Jondral, "Software Defined Radio - Basics and Evolution to Cognitive Radio," EURASIP Journal on Wireless Communications and Networking, no. 3, pp. 275-283, 2005.

[2] E. J. Candès and M. B. Wakin, "An Introduction To Compressive Sampling," Signal Processing Magazine, IEEE, vol. 25, no. 2, pp. 21-30, March 2008.

[3] R. Baraniuk, "Compressive sensing," Lecture Notes in IEEE Signal Processing Magazine, Volume 24, July 2007.

[4] M. A. Davenport, M. F. Duarte, M. B. Wakin, J. N. Laska, D. Takhar, K. F. Kelly, and R. G. Baraniuk, "The Smashed Filter for Compressive Classification and Target Recognition," Proceedings Computational Imaging $V$, vol. 6498, 2007.

[5] R. G. Baraniuk and M. B. Wakin, "Random projections of smooth manifolds," To appear in Foundations of Computational Mathematics, 2007.

[6] Z. Tian and G. Giannakis, "Compressed sensing for wideband cognitive radios," Acoustics, Speech and Signal Processing, 2007. ICASSP 2007. IEEE International Conference on, vol. 4, pp. IV-1357-IV-1360, April 2007.

[7] R. Baraniuk, M. Davenport, R. DeVore, , and M. Wakin, "A Simple Proof of the Restricted Isometry Property for Random Matrices," To appear in Constructive Approximation.

[8] J. N. Laska, S. Kirolos, Y. Massoud, R. Baraniuk, A. Gilbert, M. Iwen, and M. Strauss, "Random Sampling for Analog-To-Information Conversion of Wideband Signals," in IEEE Dallas Circuits and Systems Workshop (DCAS), 2006.

[9] J. N. Laska, S. Kirolos, M. F. Duarte, T. S. Ragheb, R. G. Baraniuk, and Y. Messoud, "Theory and Implementation of an Analog-To-Information Converter using Random Demodulation," in IEEE Int. Symp on Circuits and Systems (ISCAS), 2007. 